\title{
Management of Residual Clefts in Maxillofacial Prosthodontics: A Clinical Case
}

\section{Wijdane EL HAWARI, DMD ${ }^{1^{*}}$, Mohamed AZHARI, DMD'2, Hasnae ROKHSSI', Nadia MERZOUK, DMD and Oussama BENTAHAR, MDM³}

${ }^{1}$ Resident, Department of Prosthodontics, Faculty of Dental Medicine, University Mohammed $\mathrm{V}$, Morocco

${ }^{2}$ Assistant Professor, Department of Prosthodontics, Faculty of Dental Medicine, University Mohammed $V$, Morocco

${ }^{3}$ Professor, Department of Prosthodontics, Faculty of Dental Medicine, University Mohammed V, Morocco

*Corresponding author: Dr. Wijdane EL HAWARI, DMD, Resident, Department of Prosthodontics, Faculty of Dental Medicine, University Mohammed $V$, Rabat, Morocco

\begin{abstract}
Velopharyngeal dysfunction interest the closure of the velopharyngeal valve, it does not properly close during the production of oral sounds. It is due to velopharyngeal mislearning, velopharyngeal incompetence or velopharyngeal insufficiency.

This dysfunction is treated surgically with pharyngeal flaps or pharyngoplasties, this surgical intervention is not always successful, it can cause scarring and deficiency in mobilizing the flaps.

Indeed, when the surgery is not an indication or inadequate, the prosthetic management by soft palate obturators or palatal lift prosthesis proved successful results.

We present in this article a non-surgical approach to rehabilitation of velopharyngeal defect through a case report which illustrates the rehabilitation of a soft palate defect with a pharyngeal bulb prosthesis using a personalized technique of impression and conception of the pharyngeal bulb.
\end{abstract}

\section{Introduction}

The cleft lip and palate (CLP) constitute a frequent birth defect [1], whose management poses a real challenge for practitioners [2], this defect is resulting from the absence of fusion of the palatal fragments at the stadium of embryogenesis. The clinical manifestations of the CLP defect and velar divisions are very polymorphic [3], until this time, there is no apparent cause for the genesis of this anomaly. The probable causes are malnutrition, teratogenic and infectious agents, congenital craniofacial syndrome, head and neck cancer, or neurogenic injury $[4,5]$.

The management of CLP should respect a precise schedule which includes surgeon, speech therapist, orthodontist, maxillofacial prosthodontist [6] and psychologist; we note that there is no consensus regarding a management protocol [7].

When this defect is not managed at the adequate time or the correction was not efficient (non-operated slits or squeal slits), the practitioner can receive adults suffering from residual clefts, especially velopharyngeal dysfunctions [6].

The patients presenting this defect frequently present several symptoms such as nasal regurgitation, unintelligible speech, altered mastication and deglutition, difficulty of breathing and symptoms of the syndrome of the obstructive sleep apnea [8].

Velopharyngeal dysfunction is treated surgically with pharyngeal flaps or pharyngoplasties [9]. The surgical intervention is not always successful, it can cause scarring and deficiency in mobilizing the flaps [8].

When the surgery is not an indication or inadequate (example: Oral or systemic factors contraindicated surgical intervention [10]) the prosthetic management

Citation: EL HAWARI W, AZHARI M, ROKHSSI H, MERZOUK N, BENTAHAR O (2021) Management of Residual Clefts in Maxillofacial Prosthodontics: A Clinical Case. Int J Oral Dent Health 7:132. doi. org/10.23937/2469-5734/1510132

Accepted: June 28, 2021: Published: June 30, 2021

Copyright: (C) 2021 EL HAWARI W, et al. This is an open-access article distributed under the terms of the Creative Commons Attribution License, which permits unrestricted use, distribution, and reproduction in any medium, provided the original author and source are credited. 
by soft palate obturators or palatal lift prosthesis (PLP) has proven successful results [8].

The collaboration among the maxillofacial prosthodontic, the otolaryngologist and a speech pathologist is crucial for a correct prosthetic management of velopharyngeal dysfunctions [11].

The particularity of the case we present in this article comprises the absence of a velar substance (short and contractile veil) and transverse maxillary deficiency.

The therapeutic approach has considered these features by performing a Sheiltschy obturator and mounting prosthetic tooth with cross-bite inverse occlusion.

\section{Case Presentation}

A 47-year-old man presented to the center of consultations and dental treatments of Rabat for rehabilitation of oral cavity. The medical's history revealed that he has never got surgical operations, the reasons for consultation were: Nasal reflux, difficulty of speech, hypernasality and a rehabilitation of its bimaxillary edentulism, an extraoral examination revealed a decrease in the lower floor of his face with insufficient lip support and an aspect of mandibular retrognathism (Figure 1).

An intraoral examination revealed completely edentulous maxillary and mandibular arches; the maxillary arch was narrower than the mandibular arch with a deficient soft palate (Figure 1).

Upon intraoral examination, we found that the alveolar ridge, vestibular sulci and shape of the palate were favorable for a conventional complete denture or implant supported over denture. The patient refused implant supported over denture treatment option, we proposed it for improvement of the retention and stability of the prostheses.

We note in phonation of a prolonged " $A$ " a generalized contraction of the lateral and post walls of pharynx and a mobile and contractile velar stumps; we could describe the defect as a shortness of the uvula which not allow an adequate closure between a nasopharynx and oropharynx during function, we could classify this defect as Class II-d according to the classification of Benoist [12], the therapeutic goals were essentially the preservation of residual anatomical structures supporting the future prosthetic rehabilitation, the restoration of phonation, deglutition and chewing's functions along with ensuring the patient's physical and psychological comfort and improvement of his quality of life.

We fabricated complete denture prostheses using a conventional technique of complete denture fabrication
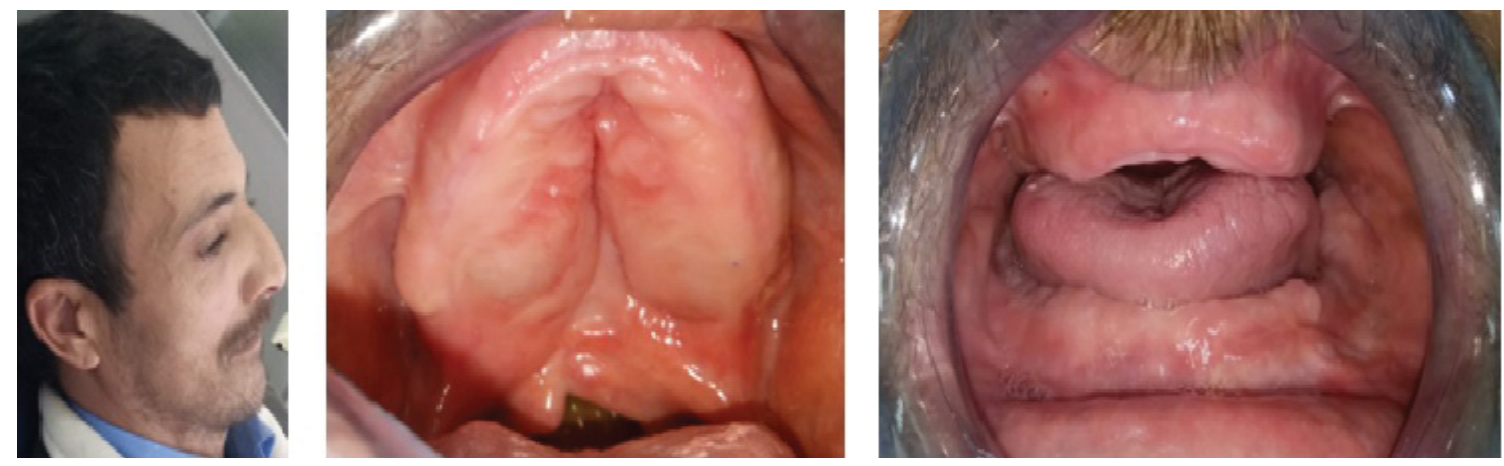

Figure 1: Extra and intraoral examination; A1) Diminished lower floor of the face and insufficient lip support; B1) Occlusal views of edentulous arches. Note deficient soft palate; C1) The maxillary arch narrower than the mandibular.
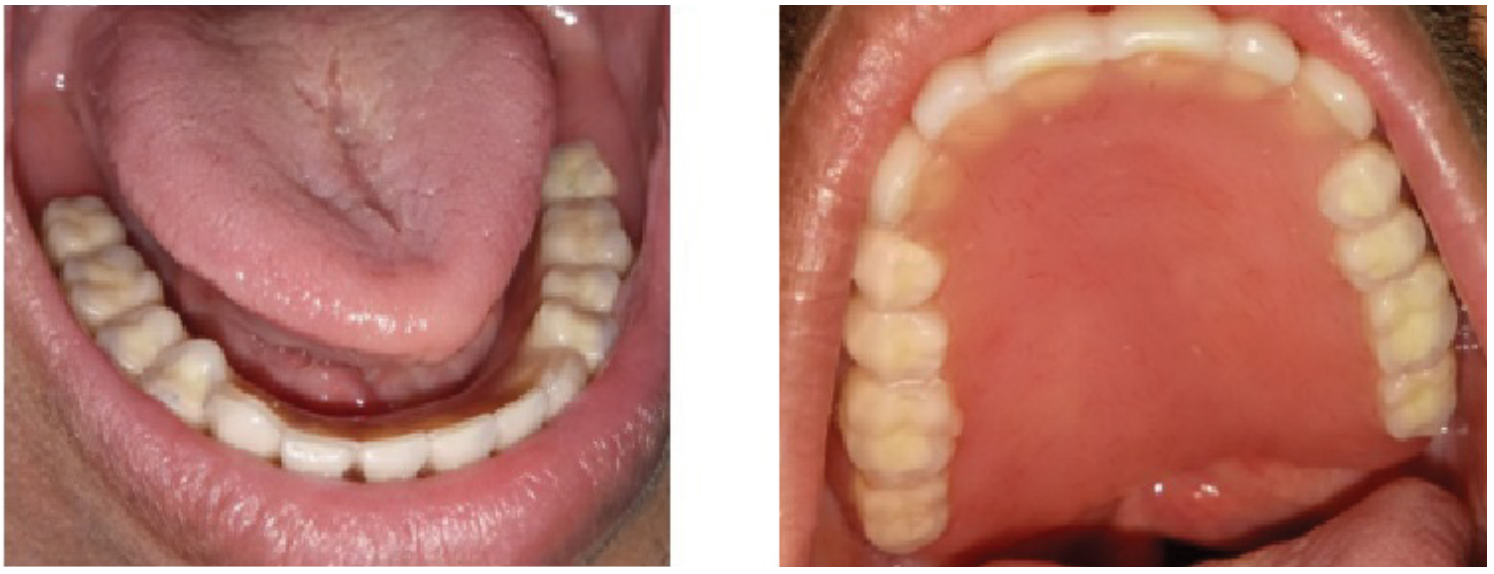

Figure 2: Intraoral view of maxillary complete denture- A cross arch arrangement. 
except for mounting tooth; we arranged the posterior teeth in cross-arch arrangement to compensate for the wider mandibular arch; this cross-arch arrangement comprises positioning the right maxillary posterior teeth on the left mandibular arch, and the left mandibular posterior teeth on the right maxillary arch (Figure 2 and Figure 3).

This technique maintains the anatomic relation and may be necessary to ensure good results when the jaw relation is abnormal [13], we delivered the prosthesis to the patient after making adequate adjustments, indeed this adaptive phase is important to judge its adaptation, stability and retention but also to involve the patient with the treatment.

We added a metallic extension at the posterior border of the prosthesis, the role of this extension is to support the impression material, it was first tried in endo-buccal to ensure that there is enough space (2-3 $\mathrm{mm}$ from all the anatomical elements in contraction), this space is utile to the impression materiel to register the contraction of velum and the pharyngeal sphincter (Figure 4); we have registered these structures by using an acrylic resin with delayed setting (Soft Liner) (Figure 4) and we have guided the patient to swallow liquid, to pronounce " $A$ " and " $O$ " and to rotate the head from side to side in different directions engaging the postural movements of the velopharyngeal structures [8].

We have used final impression light viscosity vinyl polysiloxane impression material, then we have guided patient to pronounce an occlusive phoneme such as "Pa" and "Ka" while a mirror was placed in front of the nasal orifices, this procedure allow an aim evaluation of residual nasal reflux (Figure 5).

We have seated a denture with the impression on the master cast to get the cast of the pharyngeal bulb (Figure 6), then; we performed a tutor at the posterior border of prosthesis by using a wax (Figure 6), we have converted this tutor to the metal (cobalt chrome) by using the lost-wax metal casting technique (Figure 6), then the acrylic pharyngeal bulb was processed from
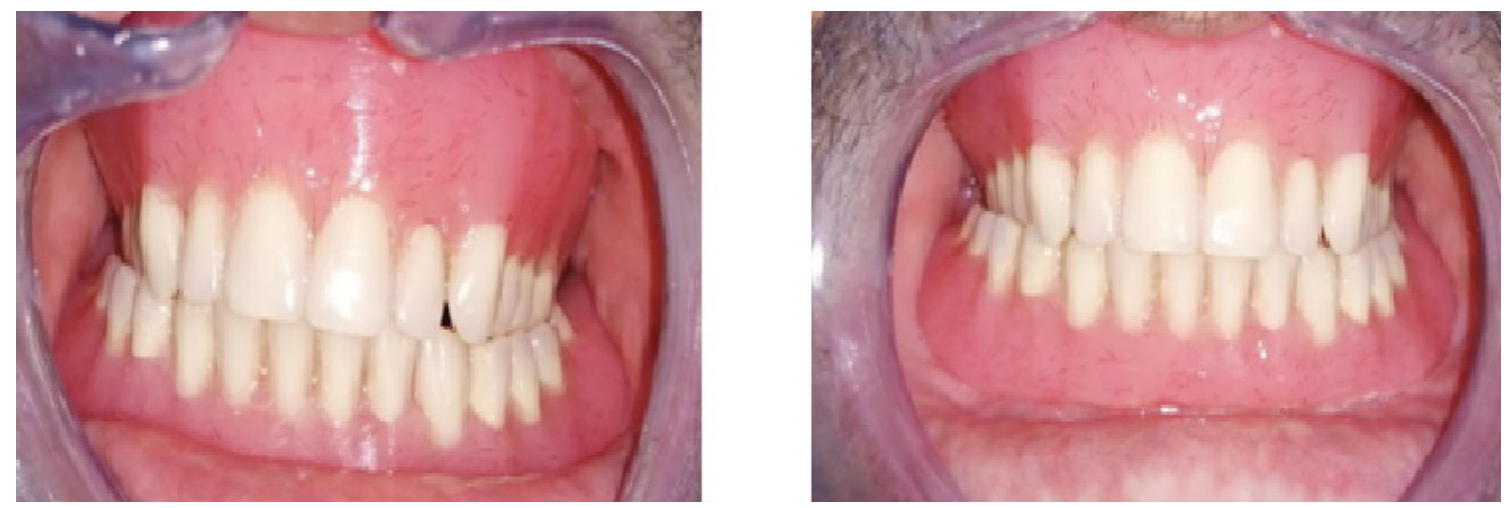

Figure 3: Intraoral views of maxillary complete denture- Inter-arch relation reversed.

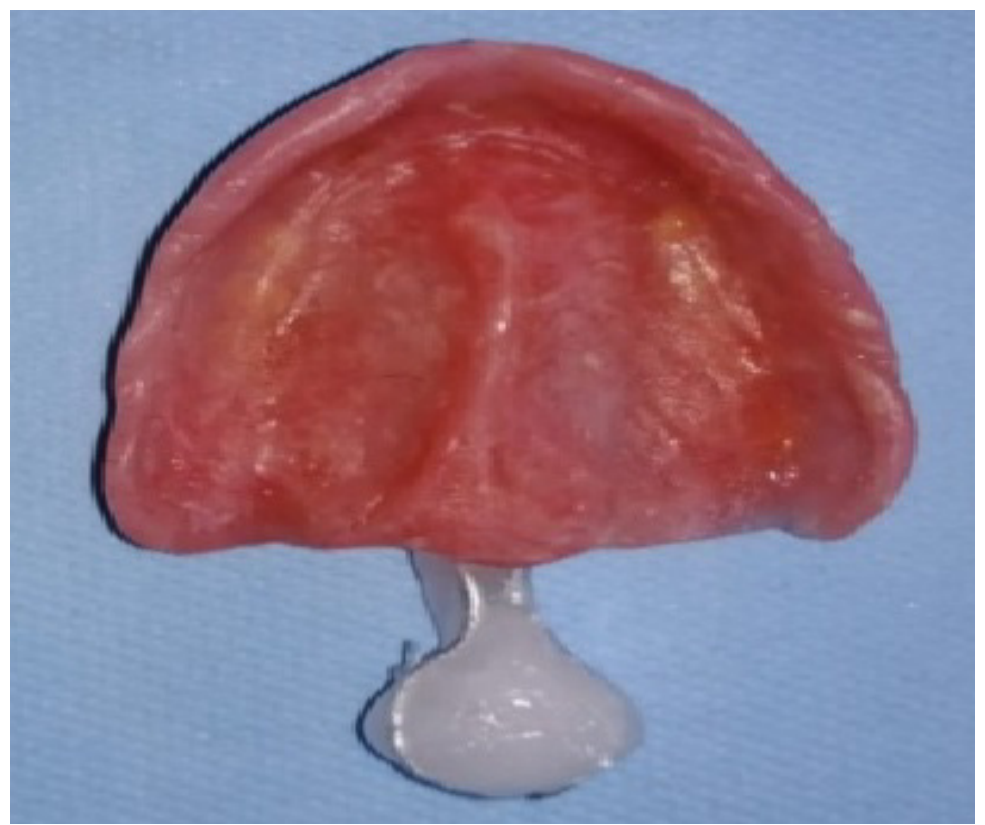

Figure 4: A view of the prosthetic intrados with a preformed stainless steel wire- Palatal obturator physiologically molded with autopolymerizing acrylic resin. 

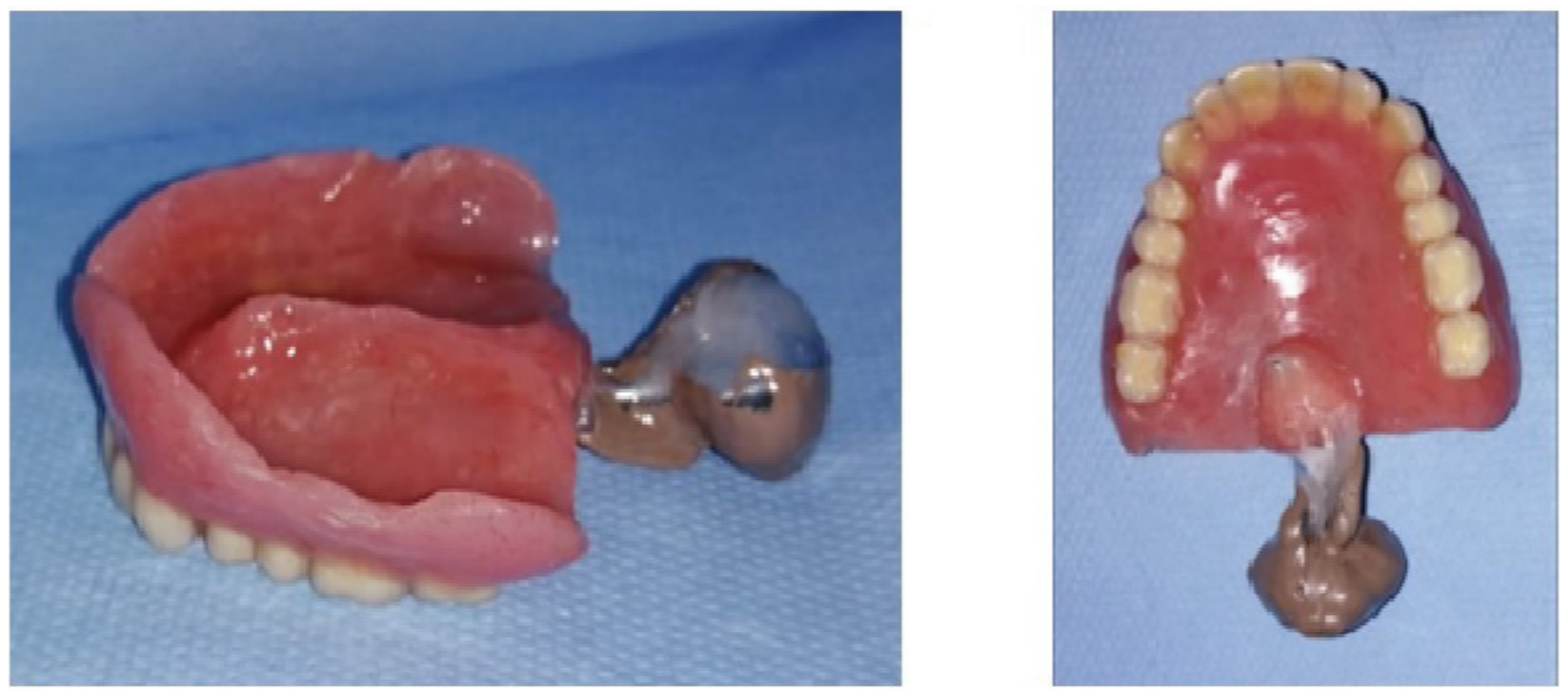

Figure 5: Final impression using medium viscosity vinyl polysiloxane impression material.
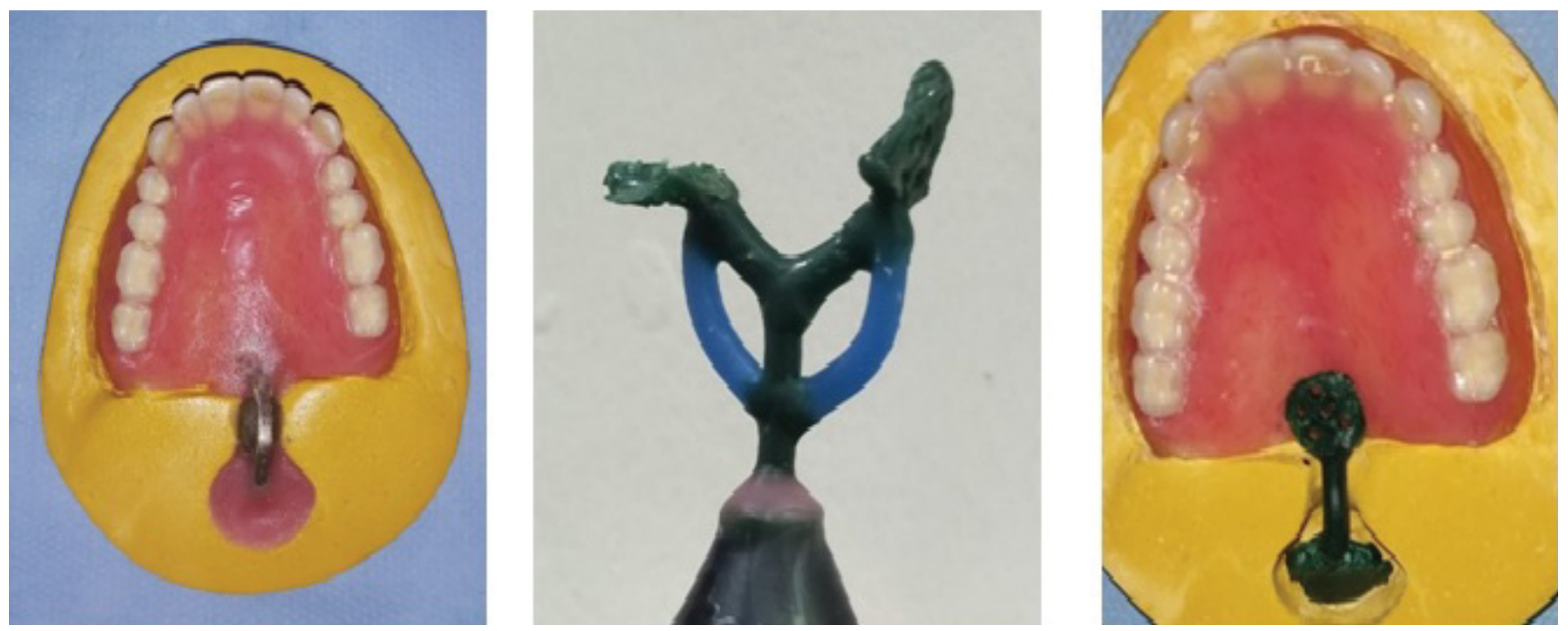

Figure 6: Making of the tutor by using the lost-wax metal casting; A6) Final cast with a wax tutor; B6) Setting up the wax tutor in the ring; C6) Final cast with a metallic tutor.

heat polymerizing acrylic resin (Figure 7).

Finally, the prosthesis comports three sections which are: Palatomaxillary anterior section, palato-alveolar middle section, nasopharyngeal posterior section (speech bulb).

We delivered the definitive prosthesis to the patient after eliminating the compression zones (Figure 7); we guided him to a speech therapist to adapt the phonation reflexes with this current device.

We carried a regular control (first 4 weeks then every month); we have not noted a nasal regurgitation, and it considerably developed the speech.

\section{Discussion}

We can describe the velopharyngeal mechanism as a muscular valve that comprises the soft palate, lateral pharyngeal walls, and the posterior pharyngeal wall [14]; it comprises contact of uvula with the posterior pharyngeal wall during swallowing and phonation.
This contact allows an adequate closure between the nasopharynx and the oropharynx; the velum elevates and moves in posterior and superior direction to close off the velopharyngeal tract during function thus, a normal accomplishment of functional speech, swallowing and breathing [8].

This mechanism permits the regulation of the amount of oral and nasal sound pressure and airflow in speech; the balance between the oral and nasal cavity in speech is important for the intelligibility of speech [5].

Prosthetic rehabilitation of soft palate defect is different according to localization and nature of defect [10], the main therapeutic aim of the management of velopharyngeal hypofunction is to restore an impervious separation between the oropharynx and the nasopharynx [15].

We note that the phonation reeducation is difficult because the patient gain compensation mechanisms from childhood [12], thus it seems to be hard to change 

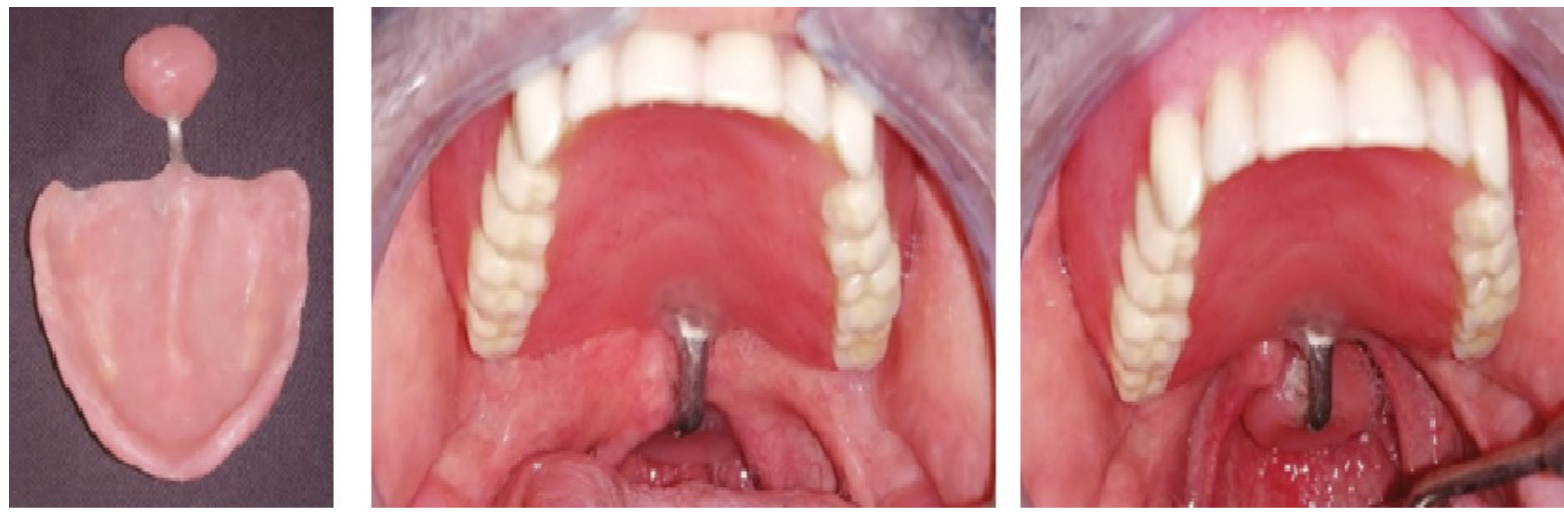

Figure 7: Maxillary complete denture with pharyngeal bulb prosthesis. A7) Acrylic pharyngeal bulb attached to denture; B7) Intraoral view of maxillary complete denture with pharyngeal bulb prosthesis without soliciting the soft palate; C7) Intraoral view of maxillary complete denture with pharyngeal bulb prosthesis in function.

these habits even with the prosthetic and surgical treatment $[14,16,17]$.

To restore this defect, there are different appliances which are adapted for each case situation; schematically, we can divide these kinds (the most used) into three types: A palatal obturator, a palatal lift and a speech bulb obturator. The palatal obturator is used to cover an open palatal defect, the palatal lift prosthesis is used to elevate the velum at its natural positions and holds it in a situation which allows a respondent contact with the posterior pharyngeal wall during function, the speech bulb obturator is an option for treatment of VP insufficiency [17], we describe this type in our clinical case; we use this appliance when the velum is short relative to the extent of the posterior pharyngeal wall; it improves speech and swallowing.

The contraction of the velopharyngeal complex is influenced by head posture [8], in effect posture [8], we used this parameter during the functional molding of the defect.

This appliance should be removal because it could be difficult sometimes to breathe and sleep with it moreover the patient should cleanse it to have a good hygiene [17], one of the pharyngeal walls should display functional mobility to achieve successful prosthetic rehabilitation according to Mazaheri and Coll [18].

Regarding materials for confection of the bulb obturator, we use the acrylic resin; we have looked off from a search literature the use of other materials, afterwards, we found that Ayliff BW and Coll. have compared the new modular silicone palatal lift prosthesis to the conventional acrylic resin design. The results of this study have shown that the new appliance reached a functional result but the conventional acrylic resin design remains superior [19].

As for the efficacy of this appliance, the study of Aboloyoun and Coll. concluded after prosthesis rehabilitation of 10 subjects studied, a significant improvement in glottal articulation, facial grimace, hyper nasality and speech intelligibility [20].

To manage this defect surgery and prosthetic fields are important for restoring the function and esthetic, it is also crucial to restore speech deficits, to increase self-esteem and psychological remedy, In this direction, the study of Norman and Coll. concluded that they need rigorous randomized controlled trials to prove an evidence base for psychological intervention techniques in patients with CLP. As for the impact of the bulb obturator on phonation, the study of A. LimaGregio and Coll. investigated nasalance and nasality at the presence of experimental openings on the speech bulb of a patient with velopharyngeal hypofunction, it concludes that different sizes of velopharyngeal influences the transfer of sound energy thus nasometry [21].

The study of DebbieSelland Coll. suggested systematic record collection, and the use of nasoendoscopy and video fluoroscopy and a blind assessment of speech outcome for an objective evaluation of speech after the prosthetic rehabilitation [22].

The prognosis of this prosthesis depends on several factors, including the age of the cleft, the history of surgery, the size of the cleft, and the associations of cleft palate and other congenital malformations [6].

We have shown through this case how to prosthetically manage velar deficit and skeletal abnormalities often associated in total toothless people with skeletal clefts, which make the originality of this case.

Concerning our patient, there was no hard palate defect, retention of conventional denture was good, we noted remarkable changes in phonetics, nasal regurgitations and social wellbeing.

At the end of our analysis, the prosthetic rehabilitation of VP defect is an adequate treatment for patients suffering from VP insufficiency and contraindicated for surgery. 
Multidisciplinary approach to prosthodontics is important for patients to reinforce their personality physically, psychologically and socially [11].

\section{Conclusion}

The prosthetic management of velopharyngeal defect is an essential option when the surgery is not indicated, the collaboration among different speakers and the motivation of the patient are crucial for the success of treatment.

\section{References}

1. Molé C, Simon E, Billiotte C, Stricker M (2010) Choix chirurgico-prothétiques dans la gestion alvéolaire des séquelles de fentes labio-maxillo-palatines. Orthod $\mathrm{Fr} 81$ : 113-126.

2. Acharya V, Brecht LE (2014) Conventional prosthodontic management of partial edentulism with a resilient attachment-retained overdenture in a patient with a cleft lip and palate: A clinical report. J Prosthet Dent 112: 117-121.

3. Destruhaut $F$, Esclassan $R$, Pomar $P$, Hennequin $A$ Vigarios-Viste $E$, et al. (2014) Approche prothétique des divisions vélo-palatines séquellaires: Classification actualisée à visée thérapeutique 165 .

4. Ayna E, Başaran EG, Beydemir K (2009) Prosthodontic rehabilitation alternative of patients with cleft lip and palate (CLP): Two cases report. Int J Dent 2009: 515790.

5. Kummer AW (2011) Communication disorders related to cleft palate, craniofacial anomalies, and velopharyngeal dysfunction. Semin Speech Lang 32: 81-82.

6. Destruhaut F, Vigarios E, Toulouse E, Pomar P (2011) Divisions vélopalatines non opérées ou séquellaires chez l'adulte: Approche fondamentale et thérapeutique en prothèse maxillofaciale. EMC.

7. Rioux E, Decker A, Deffrennes D (2012) Therapeutic thoughts on the treatment of sequellae of labial-alveolarpalatal clefts in adult patients-Part 1. Int Orthod 10: 241260.

8. Bak S-Y, Oh W-S, Erman A (2020) Treatment of velopharyngeal dysfunction: A clinical report. J Prosthet Dent 123: 888-891.

9. Pearson GD, Kirschner RE (2011) Surgery for cleft palate and velopharyngeal dysfunction. Semin Speech Lang 32: 179-190.
10. Naveen $Y G$, Jagadeesh MS, Prajapati $P$, Mallikarjuna $R$ (2013) Complete denture with pharyngeal bulb prosthesis. BMJ Case Rep.

11. Agrawal KK, Singh BP, Chand P, Patel CBS (2011) Impact of delayed prosthetic treatment of velopharyngeal insufficiency on quality of life. Indian J Dent Res 22: 356358.

12. Benoist M (1978) Réhabilitation et prothèse maxillofaciales. J Prélat.

13. Tambe A, Patil SB, Bhat S, Badadare MM (2014) Crossarch arrangement in complete denture prosthesis to manage an edentulous patient with oral submucous fibrosis and abnormal jaw relation. BMJ Case Reports.

14. Perry JL (2011) Anatomy and physiology of the velopharyngeal mechanism. Semin Speech Lang 32: 8392.

15. Rokhssi H, Azhari M, Benfdil F (2016) Prise en charge prothétique des fentes oro-faciales à l'âge adulte. A propos d'un cas Clinique 39: 11.

16. Sikka N, Jain R, Kaushik A, Rani R (2014) Prosthetic rehabilitation of a child with velopharyngeal dysfunction-A case report. J Oral Bio Craniofac Res 4: 140-143.

17. Dhakshaini MR, Pushpavathi M, Garhnayak M, Dhal A (2015) Prosthodontic management in conjunction with speech therapy in cleft lip and palate: A review and case report. J Int Oral Health 7: 106-111.

18. Mazaheri M, Mazaheri EH (1976) Prosthodontic aspects of palatal elevation and palatopharyngeal stimulation. $J$ Prosthet Dent 35: 319-326.

19. Ayliffe BW, Bressmann T, Al Mardini M, Jokstad A (2014) Evaluation of a modular palatal lift prosthesis with a silicone velar lamina for hypernasal patients. J Prosthet Dent 112: 663-671.

20. Aboloyoun AI, Ghorab S, Farooq MU (2013) Palatal lifting prosthesis and velopharyngeal insufficiency: Preliminary report. AMA 42: 55-60.

21. Lima-Gregio M, Marino VCC, Pegoraro-Krook MI, Barbosa PA, Aferri HC, et al. (2011) Nasalance and nasality at experimental velopharyngeal openings in palatal prosthesis: A case study. J Appl Oral Sci 19: 616-622.

22. Sell D, Mars M, Worrell E (2006) Process and outcome study of multidisciplinary prosthetic treatment for velopharyngeal dysfunction. Int J Lang Commun Disord 41: 495-511. 